\title{
Monte Carlo Simulation on Kinetics of Batch and Semi-batch Free Radical Polymerization
}

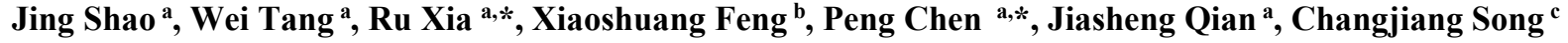 \\ aAnhui Province Key Laboratory of Environment-friendly Polymer Materials, School of Chemistry and Chemical \\ Engineering, Anhui University, Hefei 230601, China, E-mail: xiarucn@sina.com, chpecp@126.com \\ ${ }^{b}$ Division of Physical Sciences and Engineering, King Abdullah University of Science and Technology (KAUST), Thuwal, \\ 23955-6900, Saudi Arabia \\ ${ }^{\mathrm{c}}$ Anhui Zhongyi Rubber Belts Co, Ltd, Huaibei 235000, China
}

\begin{abstract}
Based on Monte Carlo simulation technology, we proposed a hybrid routine which combines reaction mechanism together with coarse-grained molecular simulation to study the kinetics of free radical polymerization. By comparing with previous experimental and simulation studies, we showed the capability of our Monte Carlo scheme on representing polymerization kinetics in batch and semi-batch processes. Various kinetics information, such as instant monomer conversion, molecular weight, and polydispersity etc. are readily calculated from Monte Carlo simulation. The kinetic constants such as polymerization rate $k_{\mathrm{p}}$ is determined in the simulation without of "steady-state" hypothesis. We explored the mechanism for the variation of polymerization kinetics those observed in previous studies, as well as polymerizationinduced phase separation. Our Monte Carlo simulation scheme is versatile on studying polymerization kinetics in batch and semi-batch processes.
\end{abstract}

Keywords: Monte Carlo simulation; free radical polymerization; semi-batch; kinetics

\section{Introduction}

Polymerization kinetics is a key issue of polymer science and engineering, which plays an important role in designing novel polymers, synthesizing polymers with narrow polydispersities (PDI), and reducing cost in industrial production of polymer etc. Various efforts have been paid on studying polymerization kinetics. In the early studies on free radical polymerization (FRP), one of the most important industrial polymerization technologies, the kinetics was treated by a simplified analytic method with assumption of the steady state approximation at very low monomer conversion stage ${ }^{[1]}$. This model is rather idealized and the kinetics at low conversion stage does not represent the full process of FRP, especially for polymerization with complex processes, such as semi-batch, ${ }^{[2]}$ telomerization $^{[3]}$ and continuous polymerization ${ }^{[4,5]}$ etc. To capture instant kinetic variables in polymerizing such as monomer conversion, molecular weight etc, the researchers have invented various online monitoring devices, such as Automatic Continuous Online Monitoring of Polymerization system (ACOMP) ${ }^{[6]}$ and Pulsed Laser initiated Polymerization in conjunction with Size-Exclusion Chromatography (PLP-SEC) ${ }^{[7]}$ etc. By using ACOMP, Wayne F. Reed and coworkers make quantitative determination of polymerization kinetics be possible, even for polymerization in a semi-batch process. ${ }^{[8]}$

Besides experimental technologies and theoretic analysing methods, computer simulation is another powerful tool for studying polymerization kinetics. As a theoretical method, computer simulation technologies may cover various polymerization processes and any elementary reactions, some of which are hard to be carried out by present experimental technologies. In addition, by involving operation condition and reaction details into the model, simulation technologies can reproduce polymerization condition much closer to practical experiment than a 
simplified theoretic analysing with ideal state assumption does.

Many simulation methodologies have been applied on studying polymerization process, which range from quantum mechanical model at atomic scale to deterministic model at industrial level by considering effects of heat and mass transfer in reactor. ${ }^{[9]}$ A commercial software Predici ${ }^{\mathrm{TM}}$ based on h-p Galerkin method has been employed by chemical engineers and experimental researchers. ${ }^{[10]}$ Among simulation methodologies, the molecular simulation technologies based on Coarse-grained Molecular Dynamics (CGMD), Monte Carlo and Dissipative Particle Dynamic (DPD) are apt to study the kinetics of FRP at micro scale. ${ }^{[1]}$ Firstly, the coarse-grained nature of molecular simulation technology make it able to simulate long polymer chains and aggregated state of polymers in solution or bulk, which is hard to be considered in a quantum mechanical model. Secondly, unlike deterministic model, the molecular simulation doesn't require prior knowledge about the reacting system, which is very versatile in studying kinetics of FRP under complex operation condition.

Pioneer works such as Akkermans and coworkers simulated polymerization process by means of molecular dynamics simulation. ${ }^{[12]} \mathrm{LV}$ and Ding introduced polymerization reaction into Monte Carlo simulation and studied kinetics of polycondensation and chain growth polymerization in concentrated solutions. ${ }^{[13-15]}$ Genzer and coworkers combined Monte Carlo motion with reaction mechanism using a probability of choosing motion or reaction and studied controlled radical polymerization in bulk and on flat surfaces. ${ }^{[16-20]} \mathrm{He}$ and coworkers studied self-condensing vinyl hyperbranched polymerization using modified Monte Carlo simulation method based on 3D bond fluctuation lattice model. ${ }^{[21-23]}$ Using the same bondfluctuation model, Lattuada and coworkers studied the kinetics of free-radical cross-linking polymerization and compared Monte Carlo simulation with experimental and numerical studies. [24] For another important molecular simulation technology, MD and DPD are also modified to study various chemical reactions. ${ }^{[25-27]}$
However, there has no systematic simulation study on FRP kinetics in semi-batch process and polymerizationinduced phase separation. Furthermore, the kinetic results proposed in molecular simulation are not deliberately evaluated by experimental data.

In this work, we introduced a Monte Carlo scheme to study kinetics of free radical polymerization in batch and semi-batch processes. By comparing the evolution of simulated polymerization kinetics under variation of polymerization conditions with those of experimental observation and Predici ${ }^{\mathrm{TM}}$ simulation, we testified the capability of our Monte Carlo routine on representing polymerization kinetics in batch and semi-batch processes. We studied the variation of kinetics parameters such as propagation constant $\left(k_{\mathrm{p}}\right)$, molecular weight $(\mathrm{Mw})$, and radius of gyration $\left(\mathrm{R}_{\mathrm{g}}\right)$ of polymers in polymerizing. The polymerization-induced self-assembly is also considered in this study.

\section{Simulation Model and Monte Carlo}

\section{Scheme}

In simulating batch and semi-batch FRP, we considered a simplified polymerization process without chain transfer and other side reactions. We employed initiation possibility $\left(P_{i}\right)$ to represent the decomposition of initiator and production of primary radicals in our Monte Carlo scheme. The elementary reactions of model FRP are those:

Initiation:

$I \stackrel{P_{i}}{\longrightarrow} I^{*} \stackrel{+M}{\longrightarrow} R^{*}$

Propagation:

$R^{*}+M \stackrel{P_{a}}{\longrightarrow} R^{*}$

Termination:

$R_{1}^{*}+R_{2}^{*} \stackrel{P_{t}}{\longrightarrow}$ Chain, or $\left(\right.$ Chain $_{1}+$ Chain $\left._{2}\right)$

The symbols in reaction equations represent the species involved in FRP, those are: I (initiator), I* (primary radical), $\mathrm{M} \quad$ (monomer), $\mathrm{R} * / \mathrm{R} 1 * / \mathrm{R} 2 * \quad$ (radicals), Chain/Chain1/Chain2 (product polymer chains). In our simulation study, reaction possibilities $P_{i}, P_{a}$ and $P_{t}$ are used 
to describe the possibilities for each elementary reactions (initiation, propagation, and termination) taking place in FRP. The reaction possibilities are different from reaction rate constants $\left(k_{i}, k_{p}\right.$ and $\left.k_{t}\right)$ in analytic equations for FRP or stochastic simulation algorithms. In FRP, termination elementary reaction may be in forms of combination or disproportionation. The former produces only one chain and the latter produces two chains (Chain 1 and Chain 2 in equation (3)).

We used a Monte Carlo scheme based on 3D bond fluctuation lattice model in NVT ensemble ${ }^{[28,29]}$ and incorporated aforementioned elementary reactions into the routine. The 3D bond fluctuation Monte Carlo technology has been widely used on studying physical properties of polymer system and also simulating kinetics of polycondensation, living/controlled polymerization, and cross-linking polymerization etc. ${ }^{[12,23]}$ Here, we just give a brief description on Monte Carlo algorithm. Being same with previous Monte Carlo simulation using 3D bond fluctuation lattice model, the coarse-grained monomer unit is modeled as a particle occupying 8 sites of a cubic lattice in simulation box. A polymer chain is composed of interconnected monomer units with five kinds of possible bond lengths $(2, \sqrt{5}, \sqrt{6}, 3, \sqrt{10})$ between neighboring units. In this study, we take initiator as a kind of monomer unit with potential of initiating and terminating polymerization. The initiator, monomer and monomer unit in a polymer chain can move randomly if there is no occupancy and energy favored by a Monte Carlo criterion. The moving vector sets and excluded volume rule are imposed to avoid bond crossing and species overlapping in Monte Carlo motion. ${ }^{[28,29]}$

To combine the reaction mechanism with Monte Carlo motion, we set a hybrid simulation routine with "selectionmotion/reaction" cycling. Firstly, a monomer unit and a set of sites at its nearest neighbor positions are randomly selected. The selected monomer unit can be an initiator, a monomer or a reacted monomer unit in a polymer chain. In case of the selected sites being vacant, a Monte Carlo "motion" is considered. In case of the sites being occupied by a monomer unit, a "reaction" judgment is carried out by analyzing the species of initially selected monomer unit and that at selected sites. If two selected monomer units can react with each other, the corresponding elementary reaction takes place under permission of its reaction possibility. Otherwise, there is nothing to do and go on with another "selection-motion/reaction" cycle. The simulation runs from a homogeneous state for initial initiators and monomers until the depletion of monomers due to polymerizing. To overcome the finite size limitation of coarse-grained simulation technology on sampling, we make a parallelization for a series of independent simulation runs. The kinetics information, such as monomer conversion, molecular weight, and polydispersity, are averaged from these independent simulation runs at the same polymerization time. In this study, we use Monte Carlo Steps to calculate polymerization time, which has nothing to do with reaction time in experiment. We here just compare the figuration and evolution of simulated kinetics curves with experimental observation and those of Predici ${ }^{\mathrm{TM}}$ simulation.

In this simulation work, all initiators and monomers in system are reactive from the beginning of polymerization for batch FRP; for semi-batch FRP, only a predetermined amount of monomers are reactive at the beginning of polymerization and then the other monomers are gradually "activated" to mimic the semi-batch process of continually injecting monomer into reacting solution in experiment.

The simulations are carried out in boxes with periodic boundary conditions applied on all directions. We set volume occupancy of occupied sites by simulated species over box sites no more than $12 \% \mathrm{vol} / \mathrm{vol}$ to mimic a solution polymerization state. In this study, we have tried simulation boxes with varied dimensions $(50 \times 50 \times 50,60 \times 60 \times 60$, and $70 \times 70 \times 70$ etc.), and parallelization of 4,8 , or 16 independent simulation runs. We found that simulation in box $50 \times 50 \times 50$ with a parallelization of 8 independent simulation runs showed depressed fluctuation and efficient sampling for polymerization kinetics information. So, the simulation results presented in this paper are carried out in 
box $50 \times 50 \times 50$ with a parallelization of 8 independent simulation runs.

\section{Results and Discussion}

Santanakrishnan and coworkers once carried out a systematic study on batch and semi-batch FRP of aqueous $\mathrm{N}$-vinylpyrrolidone (NVP) using both experimental technology and simulation by Predici ${ }^{\mathrm{TM}}$ software. ${ }^{[30]}$ In their study, kinetics data of NVP FRP are elaborate and reasonable; the Predici ${ }^{\mathrm{TM}}$ simulation results agreed well with experimental observations. To testify the capability of our Monte Carlo scheme on studying polymerization kinetics, we carried out a series of FRP simulation using the reaction conditions in their study as reference ${ }^{[30]}$ and compared our Monte Carlo simulation result with experimental and Predici ${ }^{\mathrm{TM}}$ studies.

In this study, we set polymerization possibilities as those: initiation possibility $P_{i}$ being in order of $10^{-6}$ to $10^{-4}$, propagation possibility $P_{\mathrm{a}}$ being in order of $10^{-2}$, and termination possibility $P_{t}=1.0$ with combination and disproportionation being equal with each other. Those parameters setting is corresponding to a well-known state of elementary reaction rates in FRP $k_{i}<k_{a}<<k_{t}$.

Simulation on Batch FRP. Previous experimental and theoretic studies on batch FRP have shown that initial concentrations of initiator and monomer play important effects on polymerization kinetics. For FRP of NVP in aqueous phase, with increasing of monomer concentration the polymerization rates decreases (Figure $5 \mathrm{a}^{30}$ ), in contrast the polymerization accelerate with the increase of initiator concentration (Figure $5 \mathrm{~b}^{30}$ ).

Our Monte Carlo simulation readily reproduced these phenomena as shown in Figure 1a and 2a. We also presented the variation of molecular weights (number averaged molecular weights, Mn, and Polydispersity, PDI) with increasing of initial initiator or monomer concentration in Figure $1 \mathrm{~b}$ and Figure $2 \mathrm{~b}$. The Monte Carlo simulation results are accordant with the experimental observation and
Predici ${ }^{\mathrm{TM}}$ simulation: with increasing of concentrations of initiator, the molecular weights of polymerization products drop, however, the molecular weights of products increase with increasing of initial monomer concentration (Table $3^{30}$ ). It is worthy to be mentioned that these results are comparable with the kinetics of acrylic acid polymerization. ${ }^{[31]}$
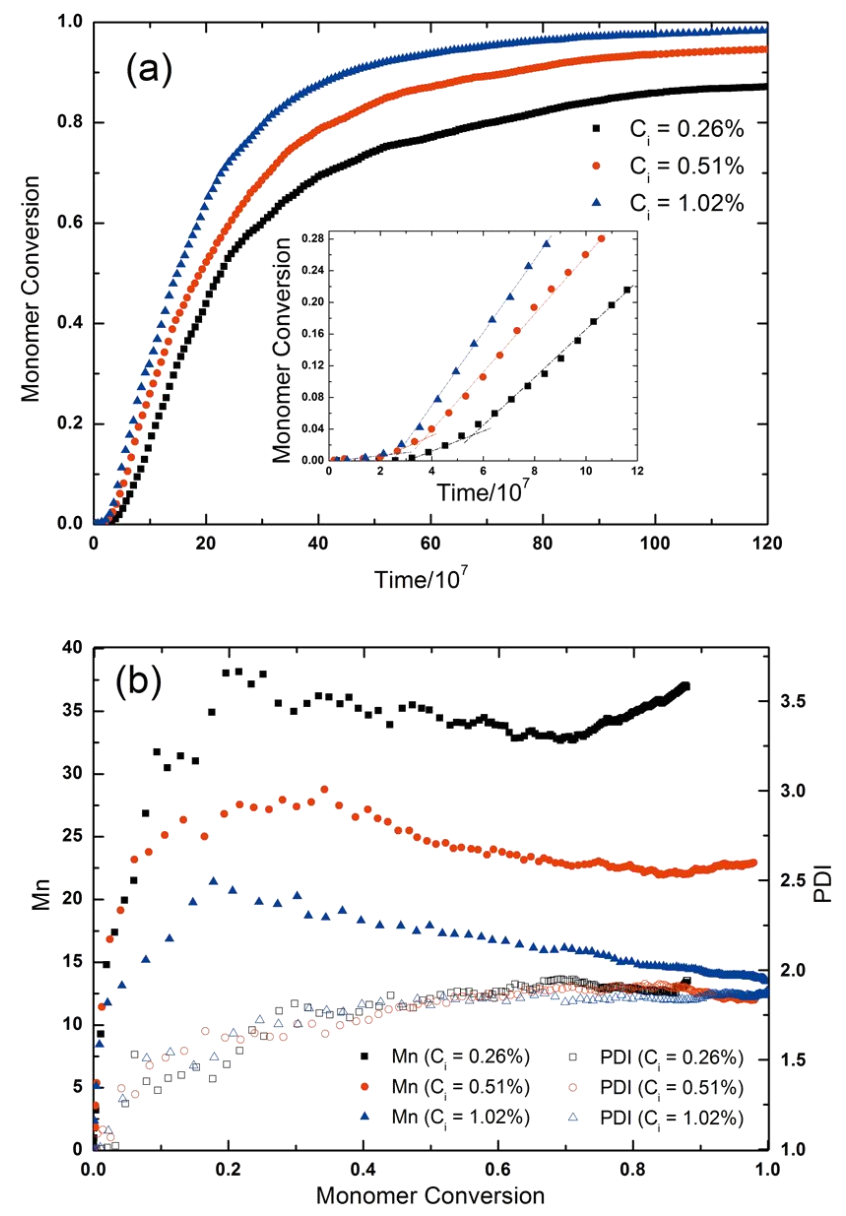

Figure 1 Monte Carlo simulation on batch FRP at relative low temperature with variation of initial initiator concentration $\left(C_{\mathrm{i}}\right) . C_{\mathrm{m}}=0.08, P_{i}=1.0 \times 10^{-6}$ $\sim 1.27 \times 10^{-4}, P_{a}=1.2 \times 10^{-2}, P_{t}=1.0$. (a) Kinetics profiles of monomer conversion versus polymerization time; (b) Kinetics profiles of Mn (PDI) versus monomer conversion.

In experiment by Santanakrichnan et al, they used a thermal initiator V-50 to initiate NVP polymerizing. ${ }^{[30]}$ According to the report, the initiation of thermal initiator $\mathrm{V}$ 50 may be retarded at the beginning of polymerization, because the residual oxygen in the system will consume some activated initiators. Although the decomposing rate of initiators is steady, the effective primary radical concentration at the beginning of polymerization is lower 
than that at middle of polymerization. As a result, the conversion curves of polymerization at low temperature (Figure $5^{30}$ ) show relative gentle slope at the beginning and then steep slope owning to the increasing of polymerization rates. The conversion curve with such evolution of polymerization looking like a "S" shape, we call it "Scurve".
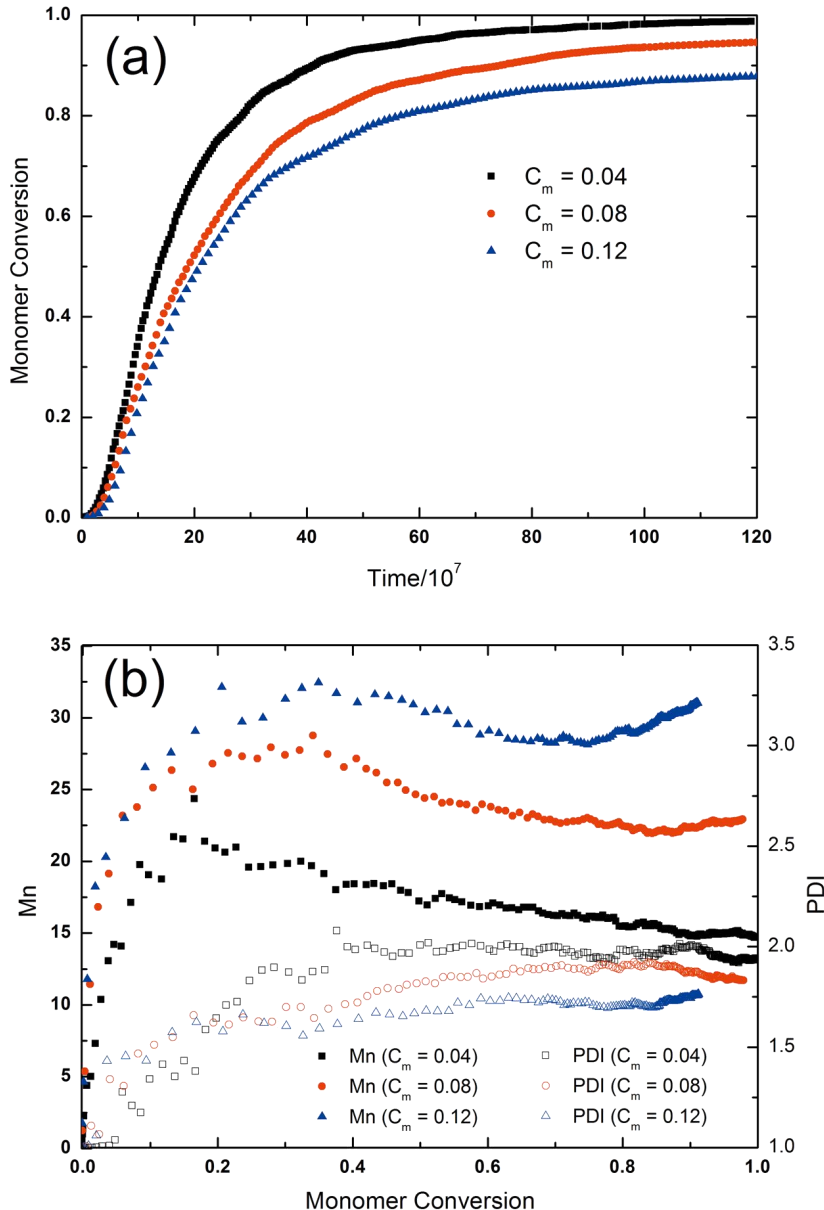

Figure 2 Monte Carlo simulation on batch FRP at relative low temperature with variation of initial monomer concentration $\left(C_{\mathrm{m}}\right) . C_{\mathrm{i}}=0.51 \%, P_{i}$ $=1.0 \times 10^{-6} \sim 1.27 \times 10^{-4}, P_{a}=1.2 \times 10^{-2}, P_{t}=1.0$. (a) Kinetics profiles of monomer conversion versus polymerization time; (b) Kinetics profiles of $\mathrm{Mn}$ (PDI) versus monomer conversion.
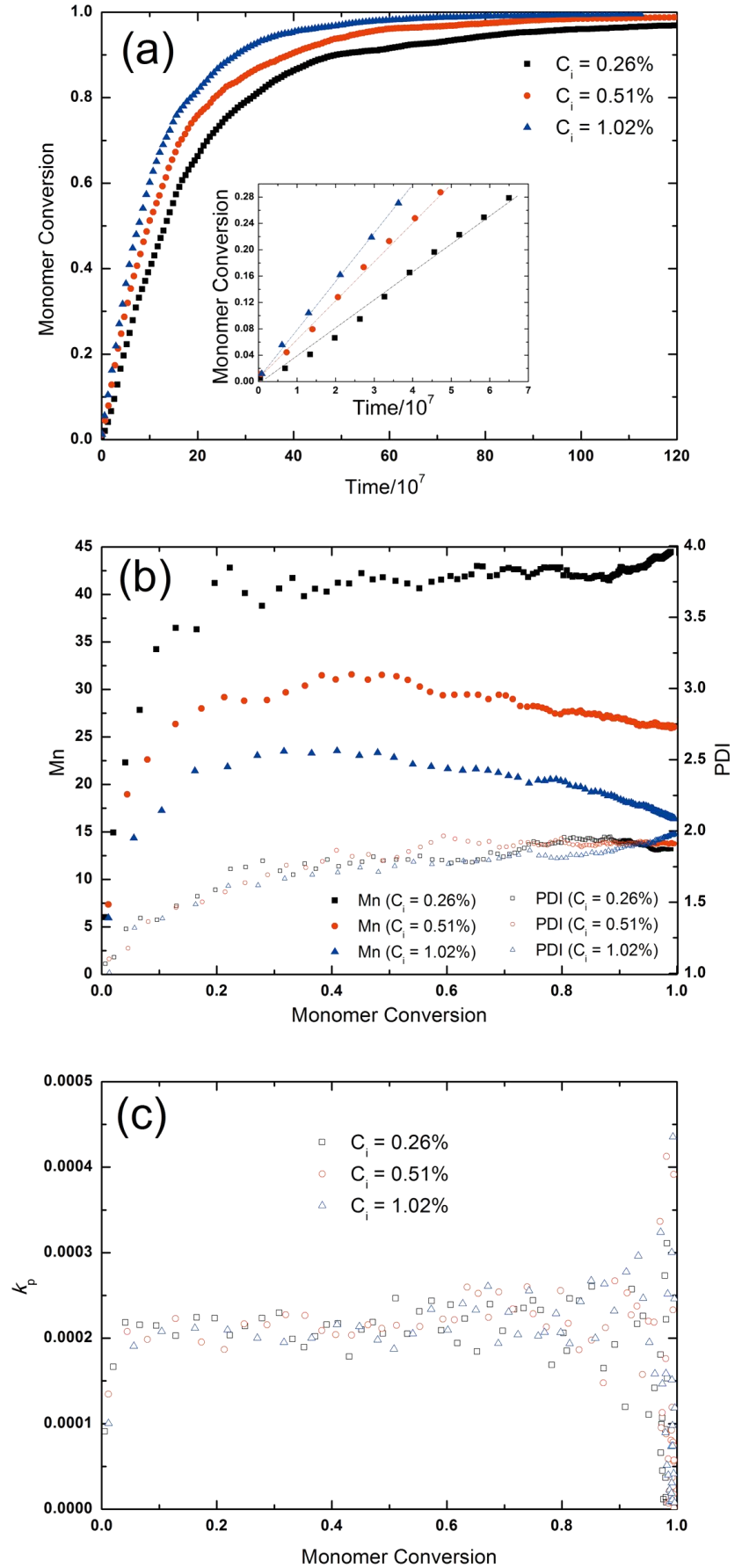

Figure 3 Monte Carlo simulation on batch FRP at relative high temperature with variation of initial initiator concentration $\left(C_{\mathrm{i}}\right), C_{\mathrm{m}}=0.08, P_{\mathrm{i}}=$ $1.99 \times 10^{-5} \sim 1.46 \times 10^{-4}, P_{\mathrm{a}}=2 \times 10^{-2}, P_{\mathrm{t}}=1.0$. (a) Kinetics profiles of monomer conversion versus polymerization time; (b) Kinetics profiles of Mn (PDI) versus monomer conversion; (c) Kinetics profiles of propagation constants $k_{\mathrm{p}}$ versus monomer conversion.

In their Predici ${ }^{\mathrm{TM}}$ simulation, ${ }^{[30]}$ Santanakrishnan and coworkers introduced inhibition reaction into the model to make simulation conversion curves fit well with those of experiments. However, the inhibition reaction in Predici 
simulation only delays the start of polymerization other than produces a "S" shape for it, their monomer conversion curves of Predici ${ }^{\mathrm{TM}}$ simulation with inhibition reaction don't start from the beginning of polymerization, where an induction period exists. In our Monte Carlo simulation, we modeled the retarding process for thermal initiator by imposing a variation of $P_{\mathrm{i}}$ from $1.0 \times 10^{-6}$ to $1.27 \times 10^{-4}$ in polymerization. This trick makes our Monte Carlo scheme be able to produce a relative low concentration of primary radical at the beginning of polymerization being similar to the condition in experiment. The monomer conversion curves in our Monte Carlo simulation exhibit " $\mathrm{S}$ " shape as shown in the inset of Figure 1a, which is same with that in experiment. It's worthy to be mentioned that to mimic a relative low polymerization temperature, we set the polymerization possibilities $P \mathrm{i}, P \mathrm{a}$ in simulation $\left(P_{i}=\right.$ $\left.1.0 \times 10^{-6} \sim 1.27 \times 10^{-4}, P_{a}=1.2 \times 10^{-2}\right)$ to be less than those $\left(P_{i}\right.$ $=1.99 \times 10^{-5} \sim 1.46 \times 10^{-4}$, and $P_{\mathrm{a}}=2 \times 10^{-2}$ ) in simulating polymerization at a high temperature.

At higher reaction temperature, the retarding on initiating is relieved owing to the fast reaction of radicals with residual oxygen in solution. We adjusted the variation of parameter $P_{\mathrm{i}}$ to being from $1.99 \times 10^{-5}$ to $1.46 \times 10^{-4}$, and $P_{\mathrm{a}}$ to be $2 \times 10^{-2}$ to mimic a condition with increased reaction rates at high temperature. The simulation results are shown in Figure 3 and 4. Again, our Monte Carlo simulation results are highly resemble with those of experimental observation and Predici ${ }^{\mathrm{TM}}$ simulation: At high reaction temperature, the initiator and monomer concentration show similar effects on polymerization rates as those at low reaction temperature, while the difference between curves of the monomer conversion versus polymerization time shrinks. As shown in the inset of Figure 3a, the "S" shapes in our Monte Carlo simulation fade away, being same with experimental result, though the setting of $P_{i}$ variation still exist in algorithm.

In our Monte Carlo simulation, we calculated $k_{p}$ values according to formula (4)

$$
k_{p}=\frac{-\frac{d \Delta C_{m}}{d t}}{C_{m} \times C_{r}}
$$
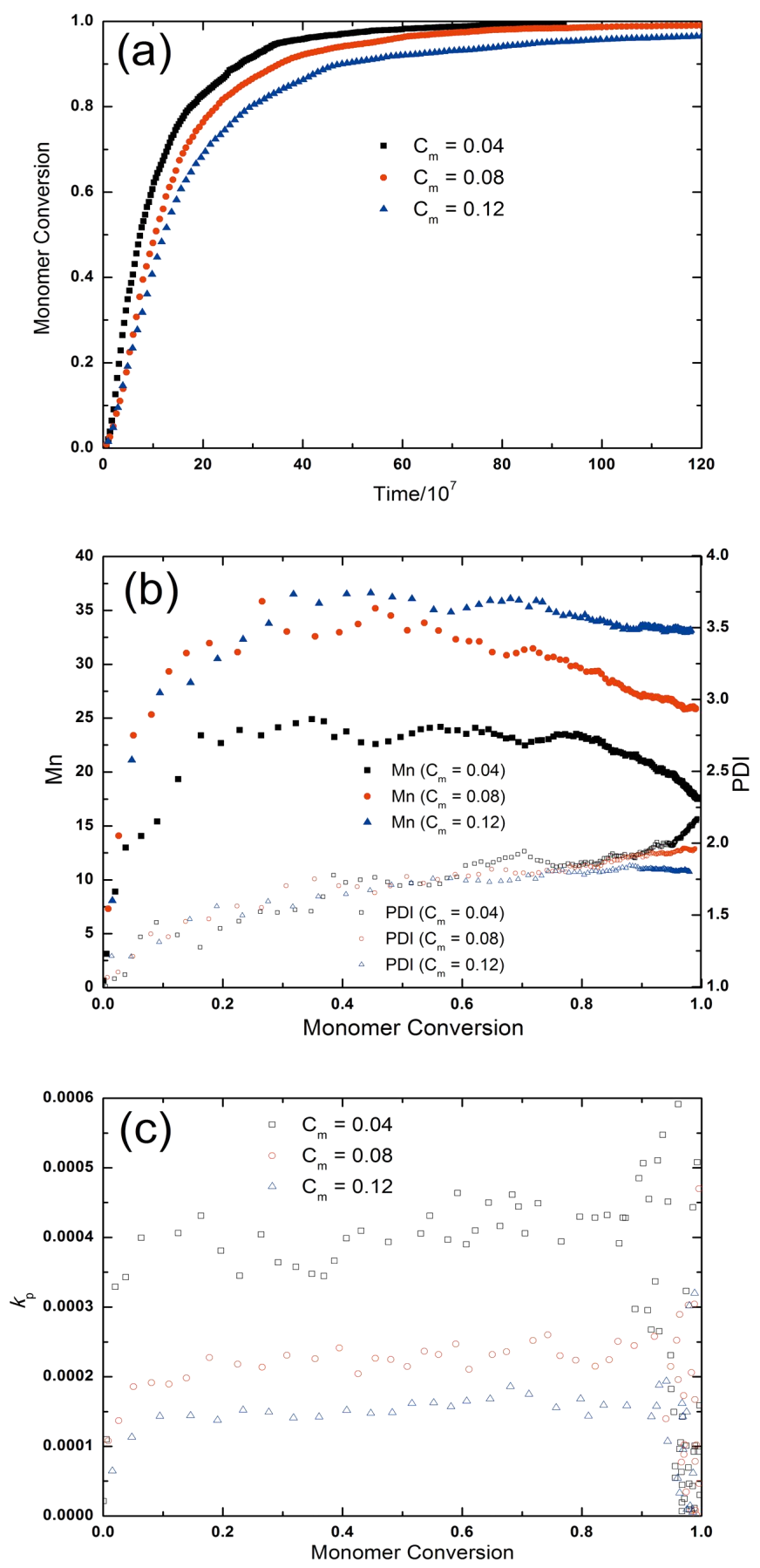

Figure 4 Monte Carlo simulation on batch FRP at relative high temperature with variation of initial monomer concentration $\left(C_{\mathrm{m}}\right) . C_{\mathrm{i}}=0.51 \%, P_{i}=$ $1.99 \times 10^{-5} \sim 1.46 \times 10^{-4}, P_{a}=2 \times 10^{-2}, P_{t}=1.0$. (a) Kinetics profiles of monomer conversion versus polymerization time; (b) Kinetics profiles of Mn (PDI) versus monomer conversion; (c) Kinetics profiles of propagation constants $k_{p}$ versus monomer conversion.

In formula (4), $C_{\mathrm{m}}$ and $C_{\mathrm{r}}$ mean the instant concentrations of monomer and radicals in system, respectively. Here, the propagation constant $k_{\mathrm{p}}$ has a unit of 
$(C \times \text { time })^{-1}$. The typical kinetics profile of propagation constant $k_{p}$ with variation of monomer conversion can be found in Figure 3c, and $4 \mathrm{c}$. According to Figure 3c, the initial concentration of initiator shows less effect on $k_{p}$ values of FRP, while the profiles of $k_{p}$ values with different initial concentration of monomer clearly distinguish from each other as shown in Figure 4c. However, except those at the beginning and end of polymerization $k_{p}$ values are less disturbed for a batch FRP process. This means the propagation rate keeps constant at most of the polymerization time in a batch FRP process. At the beginning of polymerization, $k_{p}$ values increase rapidly within a very limited range of monomer conversion (less than 0.05 in our Monte Carlo simulation). At the end of polymerization, $k_{p}$ values vibrate intensively with a tendency of dropping down.

As found in experimental studies, the propagation constant $k_{p}$ increases as initial monomer concentration decrease. ${ }^{[32]}$ It is believed that $k_{p}$ values should increase along with the monomer conversion owning to the decreasing of monomer concentration. ${ }^{[28]}$ However, our Monte Carlo simulation found the variation of $k_{p}$ in polymerizing is very complicated. We think that the effect on $k_{p}$ of initial concentration of monomer is not equivalent to that of instant concentration of monomer during polymerizing owning to the varied chemical environment at the beginning and middle stages of polymerization. Furthermore, our simulation result may explain the difference between kinetics curves obtained by Predici ${ }^{\mathrm{TM}}$ simulation and experiment in batch: with a set of reaction constants, the Predici ${ }^{\mathrm{TM}}$ simulation result fits well with experimental data at middle stage of polymerization except those at the beginning and end of polymerization. Considering the variation of $k_{p}$ values according to our Monte Carlo simulation as shown in Figure 4c, the deviation only happens at the stage of beginning and the end when $k_{p}$ varies remarkably.

In experiment, the researchers have established an expression for $k_{p}$ variation along with initial monomer concentration. ${ }^{[32]}$ We simulated a series of batch FRP with varied initial monomer concentration and calculated $k_{p}$ values with monomer conversion being $10 \%$ to get a fitting equation as shown in Figure 5. The fitting equation of $k_{p}$ versus $C_{\mathrm{m}}$ in our Monte Carlo simulation has the same expression as that from experimental researching. ${ }^{[32]}$ This means the polymerization kinetics in Monte Carlo simulation obey the same physical law as that present in experiment researching. The mismatch of parameters in two fitting equations is partially resulting from using different concentration units: in Monte Carlo simulation $C_{\mathrm{m}}$ is of $\mathrm{vol} / \mathrm{vol}$, while the monomer concentration in experiment is measured as $w \mathrm{t} \%$.

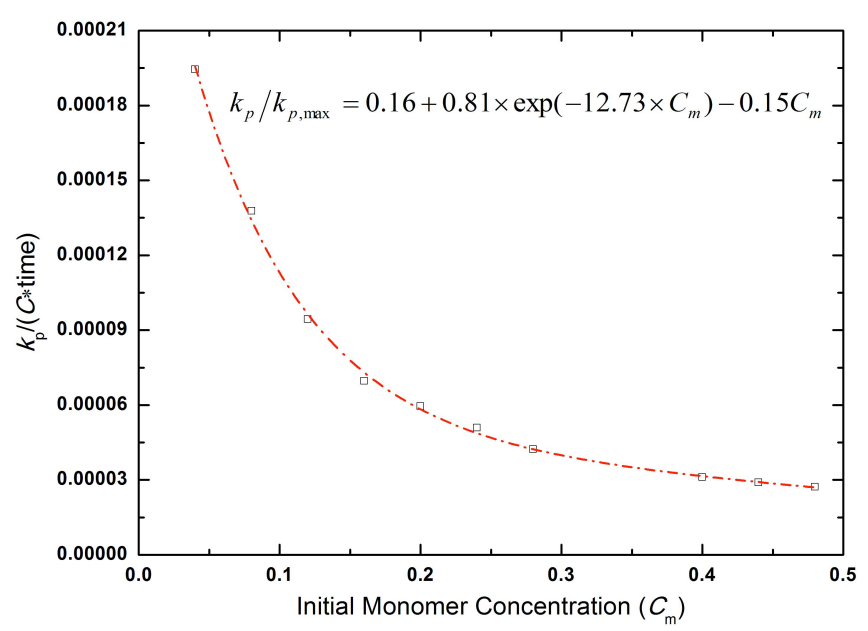

Figure 5 Monte Carlo simulated relationship of propagation constants $k_{p}$ with initial monomer concentration.

Simulation on Semi-batch FRP. In semi-batch process, monomers are continuously fed into reaction system. In Predici ${ }^{\mathrm{TM}}$ simulation, by using reaction constants $\left(k_{p}, k_{t}\right)$ according to experimentally obtained expressions, the experiment results of semi-batch FRP are well represented by simulation. ${ }^{[30]}$ In our Monte Carlo simulation on semibatch FRP, we use the same setting of reaction possibilities $\left(P_{i}=1.0 \times 10^{-6} \sim 1.27 \times 10^{-4}, P a=0.012, P_{t}=1.0\right)$ as those in batch FRP and continuously feed monomer into reaction system to mimic a semi-batch process. In Figure 6, a polymerization kinetics with two stages in monomer conversion curve is found same as those in Predici ${ }^{\mathrm{TM}}$ simulation and experiments. However, the variation of $k_{p}$ values in our Monte Carlo simulation shown in Figure 7 is different from that used in Predici ${ }^{\mathrm{TM}}$ simulation, the latter of which is relatively constant according to the description. ${ }^{[30]}$ 
In Monte Carlo simulation on semi-batch FRP, $k_{p}$ values at the beginning of polymerization is relatively high due to the very low initial monomer concentration. With increasing of monomer conversion $k_{p}$ values gradually decrease till monomer conversion being 0.8 and then the values vibrate intensively especially at the end of polymerization, which is similar with those in batch FRP. To our knowledge, the vibration of $k_{p}$ values in batch and semi-batch process are firstly reported.

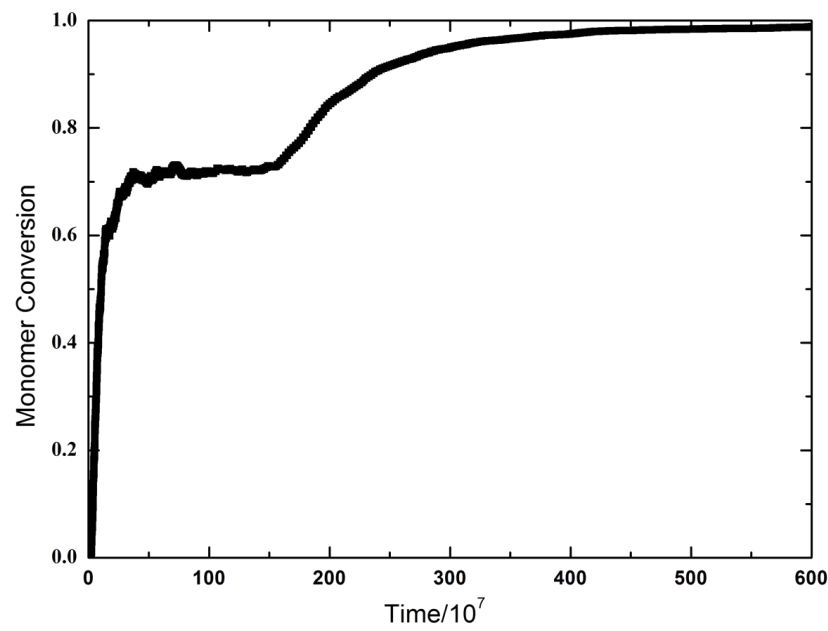

Figure 6 Monte Carlo simulated kinetics profile of monomer conversion versus polymerization time in a semi-batch FRP. $P_{i}=1.0 \times 10^{-6} \sim 1.27 \times 10^{-4}$, $P_{a}=1.2 \times 10^{-2}, P_{t}=1.0 ; C_{\mathrm{i}}=0.51 \%$, total $C_{\mathrm{m}}=0.08$ (Monomers are "fed" over a period of $170 \times 10^{7}$ ).

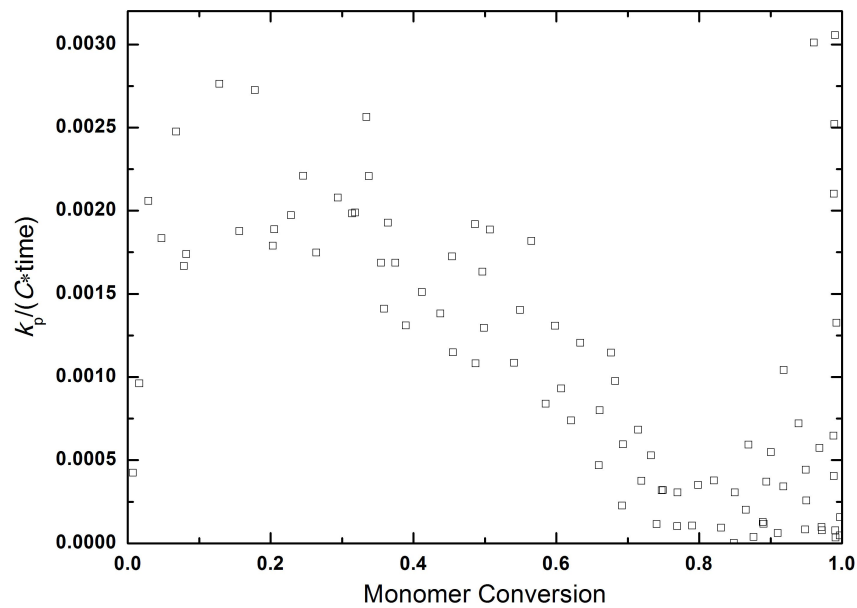

Figure 7 Kinetics profile of propagation constants $k_{p}$ versus monomer conversion in Monte Carlo simulation on semi-batch FRP. The reaction condition is same as that in Figure 6.

In experiment and Predici ${ }^{\mathrm{TM}}$ studies on semi-batch FRP, molecular weight of polymerization product increases intensively as monomer conversion approaching $1.0 .^{[30]}$ Our
Monte Carlo simulation readily reproduced such variation of molecular weights in semi-batch FRP. We found that high concentration of monomer and low concentrations of initiator and radicals, that is the high ratio of $C_{m} /\left(C_{i}+C_{r}\right)$ cause an intensive rise of molecular weights of the product.
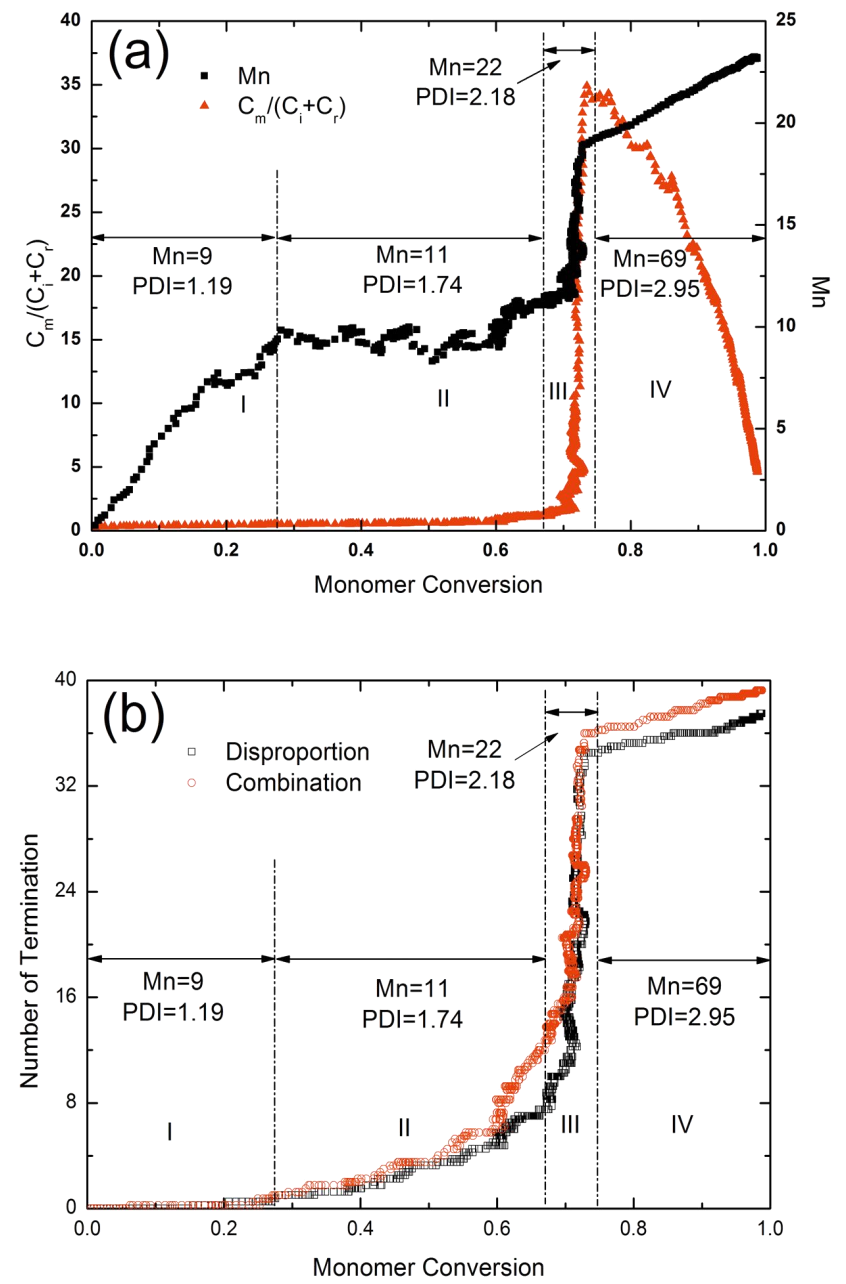

Figure 8 Variation of instant variables in Monte Carlo simulation on the semi-batch FRP. (a) Ratio of $C_{m} /\left(C_{i}+C_{r}\right)$ versus monomer conversion; (b) Number of termination versus monomer conversion.

As shown in Figure 8a, when monomer conversion is approaching 0.7 , the values of $C_{m} /\left(C_{i}+C_{r}\right)$ start to increase intensively, as a result molecular weights of polymerization product increase. We analyzed Mn and PDI for "newborn" polymers during four individual polymerization periods (I, II, III, IV shown in Figure 8). When monomer conversion increases from 0.67 to 0.75 during III period, the numbers of combination and disproportion both increase remarkably along with monomer conversion increasing (Figure $8 \mathrm{~b}$ ). The Mn of the "newborn" polymers during this period (III) is 22. 
Considering the $\mathrm{Mn}$ of total polymers increases from 11 to 19 in period III, the "newborn" polymers owning to high ratio of $C_{m} /\left(C_{i}+C_{r}\right)$ contribute most to the total Mn. During IV period, the "newborn" polymers are extreme large with $\mathrm{Mn}$ $=69$. In this period, the concentration of initiator is very low, which make primary radicals hard to be consumed and living chains hard to be terminated. As a result, Mn of polymerization products intensively increases.

\section{Simulation on Polymerization-induced Phase Separation.}

Based on molecular simulation technology, our Monte Carlo scheme is able to study phase behavior of a polymerizing system, such as spatial configuration of individual polymer chains and aggregate state of polymers at microphase. In this preliminary study, we impose attractive interaction among the segments in a polymer chain to observe polymerization-induced phase separation, which is corresponding to a precipitation polymerization in experiment. The attractive interactions are set ranging from 0 (no attractive interaction), to $0.1,0.2$ and 0.3 (strong attractive interaction).

As shown in Figure 9, attractive interaction between segments in a polymer chain shows limited influence on polymerization kinetics. There is no much difference for final monomer conversions and molecular weights of polymerization systems with different attractive interaction. However, at intermediate stage of polymerizing as monomer conversion from 0.2 to 0.9 , the polymerizing rates slightly decrease with stronger attractive interaction from 0 to 0.3 (Figure 9a), as a result, molecular weights of intermediate products drop correspondingly (Figure 9b).

The variation of polymerization kinetics under different attractive interaction can be ascribed to that polymer chains will coil up with strong attractive interaction among segments in chain, which may depress the contacting possibility of a chain radical with monomer. As shown in Figure 10 , the $R_{\mathrm{g}}$ values of polymerizing chains decrease with attractive interaction increases especially at the range of monomer conversion from 0.2 to 0.9 .
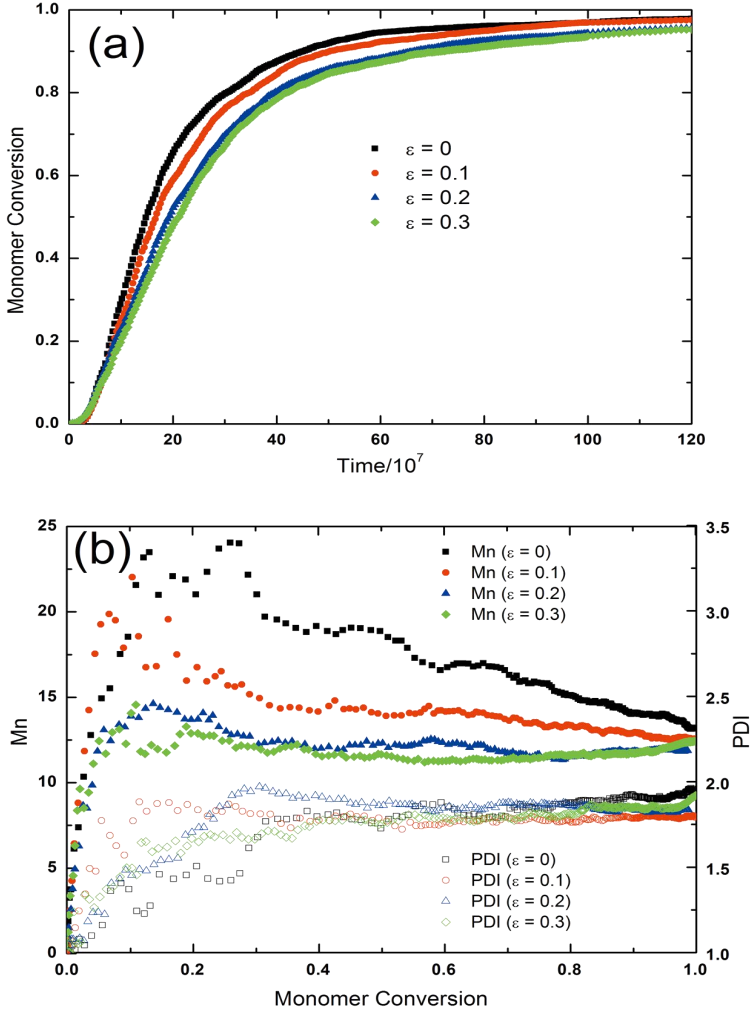

Figure 9 Monte Carlo simulation on precipitation FRP with varied attractive interaction $(\varepsilon)$ among segments in a chain. $P_{i}=1.0 \times 10^{-6} \sim$ $1.27 \times 10^{-4}, P_{a}=1.2 \times 10^{-2}, P_{t}=1.0 ; C_{\mathrm{i}}=0.51 \%, C_{\mathrm{m}}=0.04$. (a) Monomer conversion versus polymerization time; (b) Mn (PDI) versus monomer conversion.

A visual inspection on polymerization system in Figure 11 clearly shows aggregate states of polymer chains. There is no obvious phase separation for polymerization system with attractive interaction strength $\boldsymbol{\varepsilon}$ being 0 and 0.1 . With attractive interaction being strong enough, such as $\varepsilon=0.2$ and 0.3 , the polymer chains aggregate into spherical clusters. A very interesting phenomenon is that the diameters of spherical cluster decrease with $\boldsymbol{\varepsilon}$ increasing from 0.2 to 0.3 . A deep understanding on this issue should be further addressed both by experiment and simulation studies. 


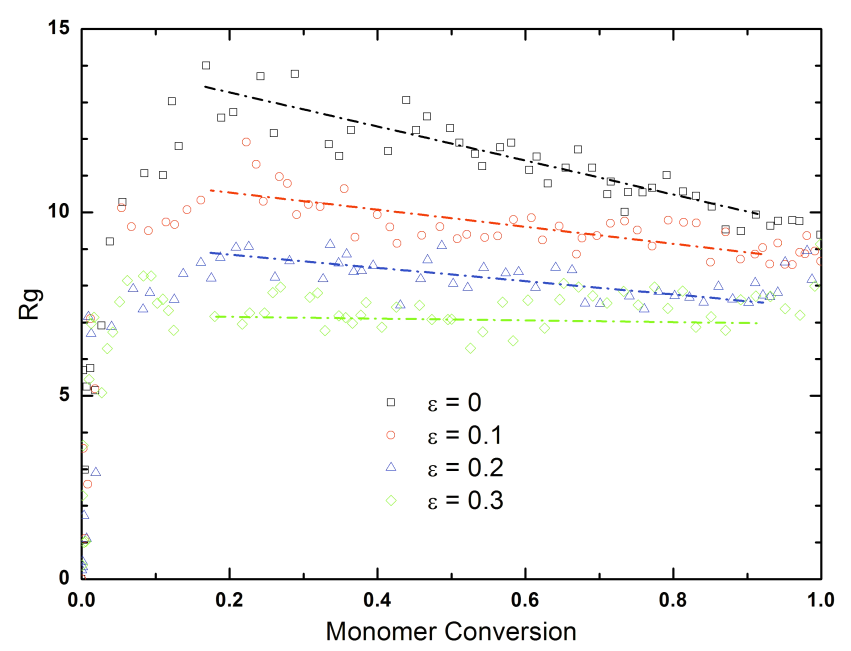

Figure 10 Radius of gyration $\left(R_{\mathrm{g}}\right)$ profiles of simulated precipitation FRP with varied attractive interaction $(\varepsilon)$. $\varepsilon=0$

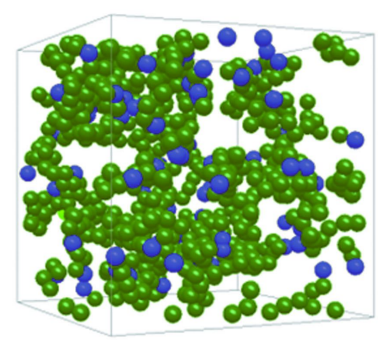

$\varepsilon=0.2$

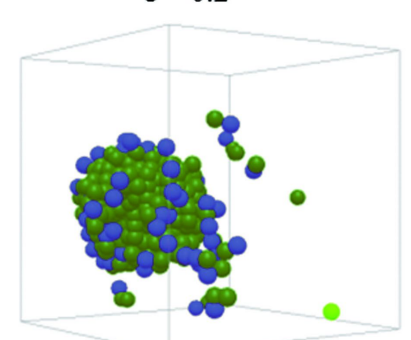

$\varepsilon=0.1$

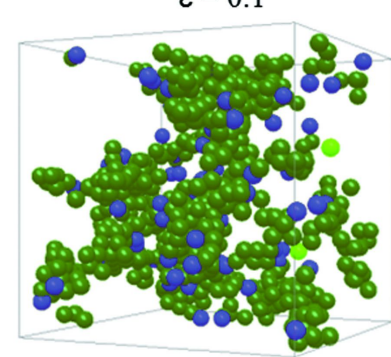

$\varepsilon=0.3$

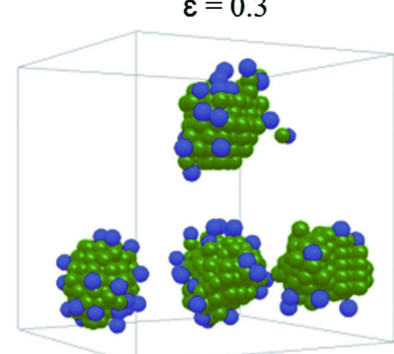

Figure 11 Polymerization-induced phase separation in Monte Carlo simulation on precipitation FRP with varied attractive interaction $(\varepsilon)$. Particles in green and blue are monomers and reacted initiators, those in light green are unreacted initiators.

\section{Conclusions}

In this study, the evolution of monomer conversion and molecular weights under different polymerization condition in batch and semi-batch FRP are well represented by Monte Carlo simulation. Our Monte Carlo scheme based on molecular simulation technology demonstrates a versatile capability on capturing physical law and polymerization kinetics in batch and semi-batch process, and it can also represent microphase state of individual and/or aggregated chains in polymerization.
For the first time, we showed that $k_{\mathrm{p}}$ values along with monomer conversion in batch and semi-batch processes vary differently. In both cases, the vibration of $k_{\mathrm{p}}$ values is rather strong at the end of polymerization. The mechanism for rising up of molecule weights in semi-batch FRP is ascribed to the variation of ratio of $C_{m} /\left(C_{i}+C_{r}\right)$, which is helpful to control the molecular weights in polymer synthesizing. The polymerization-induced phase separation is found depended on interaction strength.

There are still many questions remain to be answered, such as that the variation of $k_{p}$ values in our Monte Carlo simulation is different from that employed by a Predici ${ }^{\mathrm{TM}}$ simulation though both simulations show similar kinetics curves. The molecular simulation methods including Monte Carlo, DPD and MD etc. should find extensive applications on studying polymerization kinetics at micro scale.

\section{Acknowledgements}

This work is supported by National Natural Science Foundation of China (under Grant No. 51403001, 20804001, 20974001, 21174001, 51273001), Research Fund for the Doctoral Program of Higher Education of China (20113401110003), Natural Science Foundation of Anhui Province (1408085MKL20), "211 Project" and "Incubation Fund for Excellent Young Researcher" of Anhui University. Authors acknowledge the support from "Collaborative Innovation Center for Petrochemical New Materials (Anqing)" and "Institute of High Performance Rubber Materials \& Products" (Hefei).

\section{Reference}

(1) G. Odian, Principles of Polymerization-Fourth Edition. Wiley InterScience, Hoboken, NJ, 2004.

(2) D. Wang, X. Li, W. J. Wang, X. Gong, and B. G. Li, Macromolecules, 45, 28(2011).

(3) B. Boutevin, J Polym Sci A: Polym Chem, 38, 3235(2000).

(4) A. L. Nogueira, L. M. F. Lona, and R. A. R. Machado, J. Appl. Polym. Sci, 91, 871(2004). 
(5) J. S. Clites, H. A. Colvin, and R. D. Fiedler, Continuous polymerization process, EP Patent $0936232 \mathrm{Al}, 1999$.

(6) A. M. Alb, and W. F. Reed, Macromol. React. Eng, 4, 470(2010).

(7) S. Beuermann, and M. Buback, Prog. Polym. Sci, 27, 191(2002).

(8) A. M. Alb, and W. F. Reed, Macromolecules, 42, 8093(2009).

(9) N. Dotson, R. Galvan, R. Laurence, and M. Tirrell, Polymerization Process Modeling, 1st edition, WileyVCH, New York, 1995.

(10) M. Wulkow, Macromol. React. Eng, 2, 461(2008).

(11) D. Cuccato, E. Mavroudakis, M. Dossi, and D. Moscatelli, Macromol. Theory Simul, 22, 127(2013).

(12) R. L. C. Akkermans, S. Toxvaerd, and W. J. Briels, J. Chem. Phys, 109, 2929(1998).

(13) W. Lu, and J. Ding, Acta Chim. Sin, 63, 1231(2005).

(14) W. Lu, and J. Ding, Sci. China Ser. B, 35, 27(2005).

(15) W. Lu, and J. Ding, Macromolecules, 39, 7433(2006).

(16) J. Genzer, Macromolecules, 39, 7157(2006).

(17) M. R. Tomlinson, K. Efimenko, and J. Genzer, Macromolecules, 39, 9049(2006).

(18) S. Turgman-Cohen, and J. Genzer, Macromolecules, 43, 9567(2010).

(19) S. Turgman-Cohen, and J. Genzer, J. Am. Chem. Soc, 133, 17567(2011).

(20) S. Turgman-Cohen, and J. Genzer, Macromolecules, 45, 2128(2012).
(21) X. He, and J. Tang, J Polym Sci A: Polym Chem, 46, 4486(2008).

(22) L. Wang, and X. He, J Polym Sci A: Polym Chem, 47, 523(2009).

(23) X. Yang, L. Wang, and X. He, J Polym Sci A: Polym Chem, 48, 5072(2010).

(24) M. Lattuada, E. D. Gado, T. Abete, L. D. Arcangelis, S. Lazzari, V. Diederich, G. Storti, and M. Morbidelli, Macromolecules, 46, 5831(2013).

(25) M. Lisal, J. K. Brennan, and W. R. Smith, J. Chem. Phys, 125, 164905(2006).

(26) M. Lisal, J. K. Brennan, and W. R. Smith, J. Chem. Phys, 130, 104902(2009).

(27) H. Liu, and Z. Lu, Front. Chem. China, 6, 300(2011).

(28) I. Carmesin, and K. Kremer, Macromolecules, 21, 2819(1998).

(29) H. P. Deutsch, and R. Dickman, J. Chem. Phys, 93, 8983(1990).

(30) S. Santanakrishnan, L. Tang, R. A. Hutchinson, M. Stach, I. Lacik, J. Schrooten, P. Hesse, and M. Buback, Macromol. React. Eng, 4, 499(2010).

(31) S. S. Cutie, P. B. Smith, D. E. Henton, T. L. Staples, and C. Powell, J Polym Sci B: Polym Phys, 35, 2029(1997).

(32) M. Stach, I. Lacik, D. C. Jr, M. Buback, P. Hesse, R. A. Hutchinson, and L. Tang, Macromolecules, 41, 5174(2008). 
For the Table of Contents if it is applicable

\section{Monte Carlo Simulation on Kinetics of Batch and Semi-batch Free Radical}

\section{Polymerization}

\begin{tabular}{ll|l|l|l|l|}
\hline $\begin{array}{l}\text { A hybrid Monte Carlo } \\
\text { simulation scheme is carried out } \\
\text { to study kinetics of free radical } \\
\text { polymerization in batch and }\end{array}$ & Jing Shao, Wei Tang, Ru Xia, * \\
semi-batch processes. Various \\
kinetics data and polymerization \\
induced phase separation are \\
readily observed in simulation.
\end{tabular}

